\title{
Conductive Micropatterns Prepared by Laser-Induced Reduction of Graphene Oxide
}

\author{
Akira Watanabe ${ }^{1 *}$, Jinguang Cai ${ }^{2}$, Sayaka Ogawa ${ }^{1}$, Eiji Aoyagi ${ }^{3}$, and Shun Ito ${ }^{3}$ \\ ${ }^{1}$ Institute of Multidisciplinary Research for Advanced Materials, Tohoku University, \\ 2-1-1 Katahira, Aoba-ku, Sendai 980-8577, Japan \\ ${ }^{2}$ Institute of Materials, China Academy of Engineering Physics, \\ Jiangyou 621908, Sichuan, P. R. China \\ ${ }^{3}$ Institute for Materials Research, Tohoku University, 2-1-1 Katahira, Aoba-ku, \\ Sendai 980-8577, Japan \\ *watanabe@tagen.tohoku.ac.jp
}

\begin{abstract}
A conductive film and the micropattern were prepared by laser-induced reduction of graphene oxide (GO). A $532 \mathrm{~nm}$ laser beam was scanned on a film made from a GO dispersion solution using a galvano-scanner system. The influences of the laser irradiation conditions such as laser power, scan speed, and atmosphere on the chemical structure changes of a reduced graphene oxide ( $\mathrm{rGO}$ ) were studied by micro-Raman spectroscopy. The Raman scattering intensities of $\mathrm{D}, \mathrm{G}$, and 2D bands were remarkably influenced by the laser irradiation conditions. The formation of a few-layer graphene plane structure was suggested by a low ratio of intensities of $\mathrm{D} / \mathrm{G}$ bands $\left(I_{\mathrm{D}} / I_{\mathrm{G}}\right)$ and the shape of $2 \mathrm{D}$ band in the Raman spectra. The surface resistivity was decreased with increasing in the laser power corresponding to the decrease of the $I_{\mathrm{D}} / I_{\mathrm{G}}$ ratio in the laser-induced reduction under an inert gas (Ar) atmosphere. Conductive rGO micro-grid structures were fabricated by laser direct writing using a GO film in Ar.
\end{abstract}

Keywords: Graphene oxide, Reduced graphene oxide, Laser-induced reduction, Laser direct writing, Conductive grid structure

\section{Introduction}

The additive manufacturing and on-demand processing are important key technologies for the innovation in electronics manufacturing processes toward Internet of Things (IoT) devices, where such features as transparent, flexible and lightweight are important. The printed electronics based on solution processes has been studied widely as an innovating technology $[1,2]$. On-demand processing such as inkjet printing, 3D printing, laser direct writing etc. can be applied in the printed electronics instead of conventional manufacturing processes based on a high-energy vacuum process. Various kinds of inks prepared from nano-materials such as metal nanoparticles are used in the printing processes. We have studied the laser direct writing using Ag, $\mathrm{Au}$, and $\mathrm{Cu}$ nanoparticle inks to form conductive micropatterns and interconnection. The laser di- rect writing method has advantages in spatially selective, high speed and low temperature additive manufacturing, and it also enables the on-demand processing [3-5]. The laser direct writing can be also applied to the formation of a conductive carbon micropattern as an electrode of in-plane micro-supercapacitor (MSC) by the

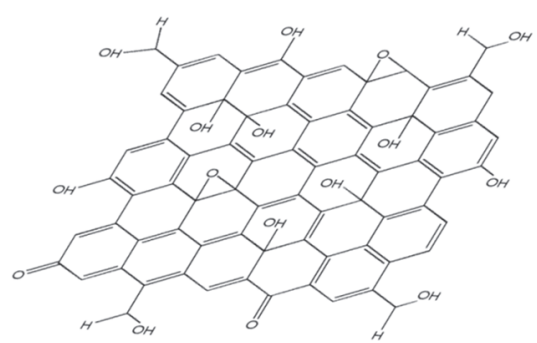

Fig. 1. Image of the chemical structure of graphene oxide. 
carbonization of polymers [6-9]. In this paper, we report the formation of conductive films by laser-induced reduction of graphene oxide (GO) (Fig. 1), and the micropatterning via laser direct writing. The laser-induced reduction of GO gives a conductive reduced graphene oxide (rGO) $[10,11]$. Although GO can be applied to various kinds of functional devices, for example, sensors, MSC and so on, the influences of laser irradiation conditions on the chemical structural changes and the electronic properties are still not clear. We studied the influences of the laser irradiation conditions such as laser power, scan speed, and atmosphere on the chemical structure and resistivity changes of the rGO.

\section{Experimental}

Few-layer GO powders (Beijing Ding Sheng Xiong Di Technology Co., Ltd.) were dispersed in water to form a GO dispersion. The solution was ultra-sonicated in a water bath. A GO film was prepared on a PET film by doctor-blade method. The thickness of the GO coating film was determined to be $0.78 \mu \mathrm{m}$ by light interferometry. The laser direct writing was performed using a galvano-scanner system consisting of a galvano-scanner head, marking card (BJJCZ EzCAD card), and a $532 \mathrm{~nm}$ CW DPSS laser (MGL-H-532 nm-1 W, CNI). The laser beam was focused through a $\mathrm{f}-\theta$ lens with the focal length of $100 \mathrm{~mm}$. Raman spectra were measured on a micro-Raman spectrometer equipped with an optical microscope (Olympus BX51), a $532 \mathrm{~nm} \mathrm{CW}$ DPSS laser, a Peltier-cooled CCD camera (DV401, Andor Technology), and a monochromator (MS257, Oriel Instruments Co.). Surface resistivity was measured by four-point probe method. The transmission spectra were observed by a UV-Vis-NIR spectrophotometer (V-670, JASCO). X-ray photoelectron spectroscopy (XPS) was performed by PHI5600 (ULVAC- PHI, Inc.).

\section{Results and discussion}

3.1. Laser-induced reduction of graphene oxide

The structural change of a GO by laser-induced reduction was studied by micro-Raman spectroscopy. The Raman spectrum of a GO before laser irradiation is shown in Fig. 2. The ratio of intensities of $\mathrm{D}$ and $\mathrm{G}$ bands is related to the $\mathrm{sp}^{2}$ domain size of graphitic materials $[12,13]$.

In our previous study on the laser induced reduction of a GO [14], a laser direct writing sys-

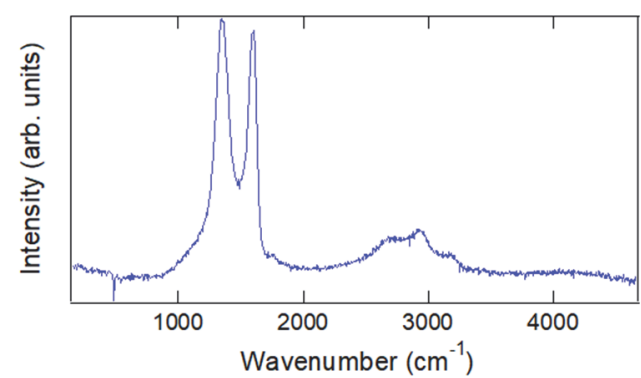

Fig. 2. Raman spectrum of graphene oxide.
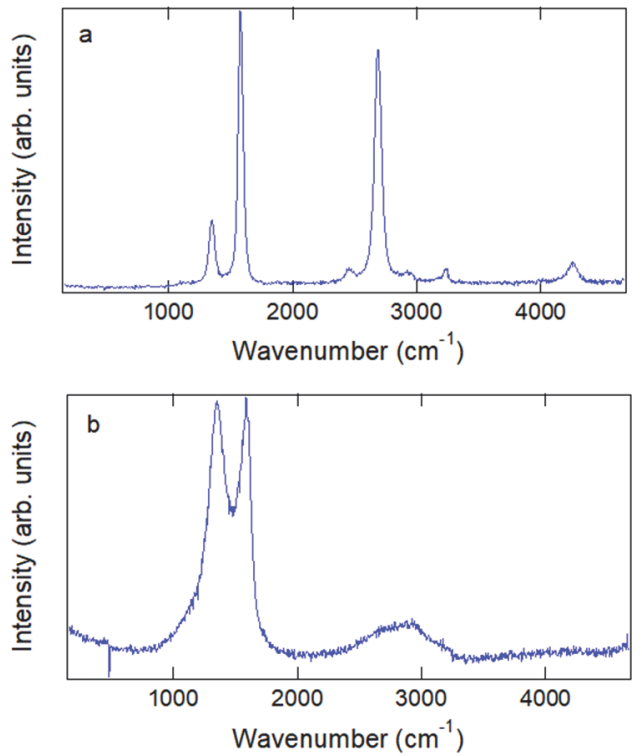

Fig. 3. Raman spectra of rGOs obtained by $532 \mathrm{~nm}$ laser irradiation in Ar under conditions of (a) laser power $600 \mathrm{~mW}$, scan speed $100 \mathrm{~mm} / \mathrm{s}$ and (b) laser power $60 \mathrm{~mW}$, scan speed $10 \mathrm{~mm} / \mathrm{s}$.

tem consisting of a CW $405 \mathrm{~nm}$ blue-violet semiconductor laser and a stepping motor-driven xyz-stage was used, where the scan speed of the laser beam was $5 \mathrm{~mm} / \mathrm{s}$. The influence of the laser beam scan speed was studied in this paper using a galvano-scanning system. The Raman spectra of the rGOs laser-reduced at different scan speeds are compared in Fig. 3, where (a) and (b) show the Raman spectra of rGOs obtained at the laser power and scan speed of $600 \mathrm{~mW}-100 \mathrm{~mm} / \mathrm{s}$ and $60 \mathrm{~mW}-10 \mathrm{~mm} / \mathrm{s}$, respectively. In both cases, the laser fluence, which is defined as the time average energy delivered per unit area, is same. The Raman spectrum of the rGO obtained under the conditions of laser power $600 \mathrm{~mW}$ and scan speed $100 \mathrm{~mm} / \mathrm{s}$ showed the remarkable decrease of the ratio of intensity of $\mathrm{D} / \mathrm{G}$ bands $\left(I_{\mathrm{D}} / I_{\mathrm{G}}\right)$ accompanying the narrowing of the Raman band. This result suggests the increase of the $\mathrm{sp}^{2}$ do- 
main size of graphitic structure. The ratio of intensities of $2 \mathrm{D} / \mathrm{G}$ bands $\left(I_{2 \mathrm{D}} / I_{\mathrm{G}}\right)$ is related to the number of graphene layer [15]. The position and the shape of the 2D band are also correlated to the number of graphene layer [16]. It has been reported that a single layer graphene shows the $2 \mathrm{D}$ Raman band centered at $2680 \mathrm{~cm}^{-1}$, which can be fitted with a single Lorentzian curve for $532 \mathrm{~nm}$ excitation [17]. The 2D Raman bands of GO and rGOs obtained by $532 \mathrm{~nm}$ laser irradiation in Ar are compared in Fig. 4. The rGO obtained under the conditions of laser power $600 \mathrm{~mW}$ and scan speed $100 \mathrm{~mm} / \mathrm{s}$ showed a single and sharp 2D peak around $2680 \mathrm{~cm}^{-1}$, which suggests that a few-layer graphene plane structure is kept even after the laser-induced reduction of the GO. The crystalline phase of the $\mathrm{rGO}$ was observed by the transmission electron microscope (TEM) images and the diffraction pattern.

The laser scan speed influenced significantly on $I_{\mathrm{D}} / I_{\mathrm{G}}$ and $I_{2 \mathrm{D}} / I_{\mathrm{G}}$ ratios as shown in Fig. 3. With decreasing in the laser scan speed from $100 \mathrm{~mm} / \mathrm{s}$
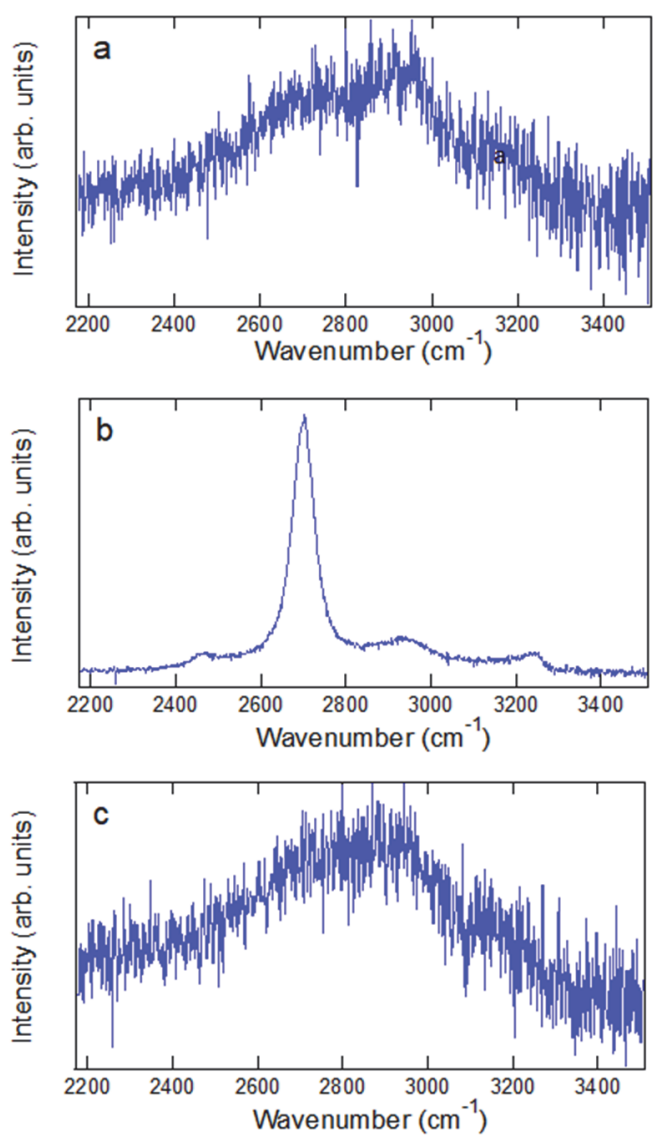

Fig. 4. 2D Raman bands of GO and rGOs obtained by $532 \mathrm{~nm}$ laser irradiation in Ar under conditions of (a) GO, (b) rGO: laser power $600 \mathrm{~mW}$, scan speed $100 \mathrm{~mm} / \mathrm{s}$, and (c) rGO: laser power $60 \mathrm{~mW}$, scan speed $10 \mathrm{~mm} / \mathrm{s}$. to $10 \mathrm{~mm} / \mathrm{s}$, the $I_{\mathrm{D}} / I_{\mathrm{G}}$ and $I_{2 \mathrm{D}} / I_{\mathrm{G}}$ ratios were not so changed compared with those of $\mathrm{GO}$ although the $I_{\mathrm{D}} / I_{\mathrm{G}}$ ratio was slightly decreased. The $I_{\mathrm{D}} / I_{\mathrm{G}}$ and $I_{2 \mathrm{D}} / I_{\mathrm{G}}$ ratios were correlated with the resistivities of rGO film. The surface resistivities of rGO films obtained at the scan speed of 100 and 10 $\mathrm{mm} / \mathrm{s}$ were 57 and 467 , respectively. When the atmosphere during laser scanning was changed from Ar to air, the degree of the laser-induced reduction judging from the changes of $I_{\mathrm{D}} / I_{\mathrm{G}}$ and $I_{2 \mathrm{D}} / I_{\mathrm{G}}$ ratios was lowered. This result suggests that the oxidation during laser irradiation may be caused by the residual oxygen even under Ar flow condition in the case of the slower laser scanning, which prevented the laser-induced reduction of the GO. The XPS analysis of rGO films confirmed it. The atomic concentration of oxygen in rGO was increased from 7.27 to $10.4 \%$ with decreasing in the laser scan speed from 100 to 10 $\mathrm{mm} / \mathrm{s}$.

\subsection{Formation of reduced graphene oxide grid}

The rGO micro-grid structures were fabricated by laser direct writing toward a transparent conductive film. Figure 5 shows the optical microscope images of rGO grid patterns before and after etching of unirradiated GO layer. The unirradiated parts of GO film can be re-dispersible in water even after drying and laser direct writing. The adhesive strength between the laser-written rGO grid structure and PET film was enough, which enabled to remove most of GO from the grid structure by wiping using a cotton swab in
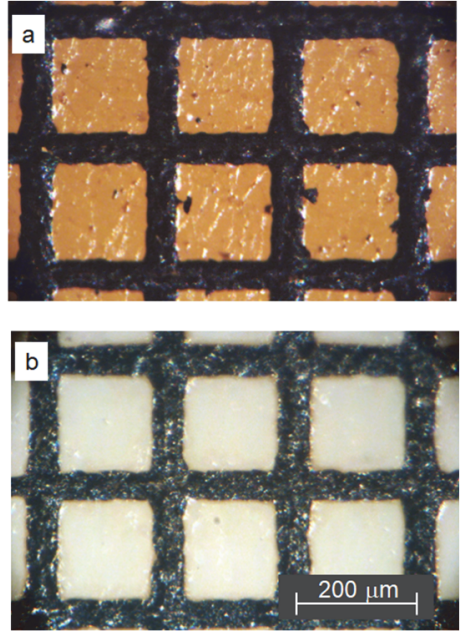

Fig. 5. Optical microscope images of rGO grid patterns before (a) and after (b) etching of unirradiated GO layer. Laser irradiation conditions: laser power $300 \mathrm{~mW}$, scan speed $100 \mathrm{~mm} / \mathrm{s}$. 
water. The bare surface of the PET film is seen in Fig. 5b. Transmission spectra of rGO grid patterns prepared at various laser powers are shown in Fig. 6. The transmittance of the rGO grid structure was increased with decreasing in the laser power because of the narrowing of the rGO line width. The rGO grid structures showed almost no wavelength dependence in visible region. The surface resistivities of rGO grid structures measured by four-points prove method were 2.48 , 4.16. $9.20,18.1$, and $41.4 \mathrm{k} \Omega / \mathrm{sq}$ at the laser power of $600,420,300,180$, and $120 \mathrm{~mW}$, respectively.

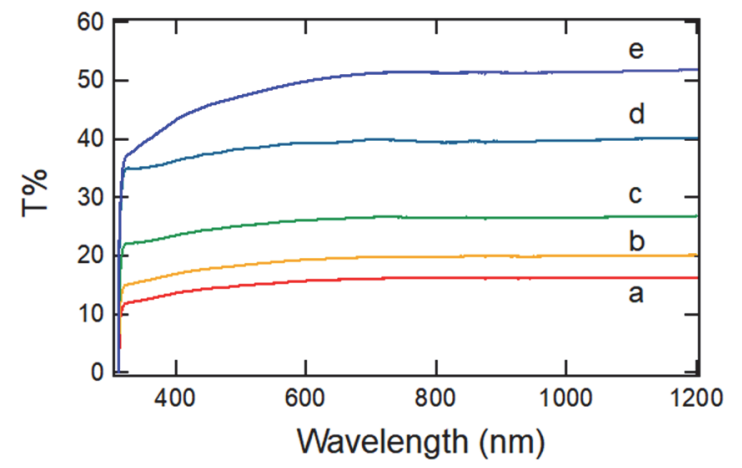

Fig. 6. Transmission spectra of rGO grid patterns after removing of unirradiated GO layer. Laser powers: (a) 600, (b) 420, (c) 300, (d) 180, and (e) $120 \mathrm{~mW}$. Scan speed $100 \mathrm{~mm} / \mathrm{s}$.

\section{Conclusion}

The laser-induced reduction of GO film was studied by using a $532 \mathrm{~nm}$ CW DPSS laser combined with a galvano-scanner system to discuss the influences of laser scan speed on the structures and properties of rGO films. In the micro-Raman spectrum measurements, the remarkable decrease of the $I_{\mathrm{D}} / I_{\mathrm{G}}$ ratio and the increase of the $I_{2 \mathrm{D}} / I_{\mathrm{G}}$ ratio were observed with increasing in the laser scan speed. The decrease of the surface resistivity was also observed with increasing in the laser scan speed showing the correlation with the changes of the Raman bands. The influence of the atmosphere during laser scanning and XPS analysis suggested that the oxidation due to the oxygen residue under Ar flow condition was lowered with increasing in the laser scan speed. A conductive and transparent rGO micro-grid structure was fabricated by laser direct writing on a GO-coated PET film.

\section{Acknowledgement}

This work was supported by a Grant-in-Aid for Scientific Research on Innovative Areas "New Polymeric Materials Based on Element Blocks (No.2401)" (JSPS KAKENHI Grant Number JP24102004), JSPS KAKENHI Grant Number JP15H04132, National Natural Science Foundation of China (No. 21603201), and China Academy of Engineering Physics (item no. TP201302-3).

\section{References}

1. I. M. Hutchings and D. G. Martin, Eds. Inkjet Technology for Digital Fabrication, Wiley (2012).

2. P. Rosa, A. Câmara, and C. Gouveia, Open J. Internet Of Things (OJIOT), 1 (2105) 16.

3. G. Qin and A. Watanabe, J. Nanopart. Res., 16 (2014) 2684.

4. G. Qin, L. Fan, and A. Watanabe, J. Mater. Proc. Technol., 227 (2016) 16.

5. G. Qin, A. Watanbe, H. Tsukamoto, and T. Yonezawa, Jpn. J. Appl. Phys., 53 (2014) 096501.

6. J. Cai, C. Lv, and A. Watanabe, J. Mater. Chem. A, 4 (2016) 1671.

7. J. Cai, C. Lv, and A. Watanabe, Nano Energy, 30 (2016) 790.

8. J. Cai, C. Lv, and A. Watanabe, RSC $A d v$. 7 (2017) 415.

9. J. Cai, C. Lv, and A. Watanabe,. ACS Appl. Mater. Interfaces, 10 (2018) 915.

10. W. Gao, N. Singh, L. Song, Z. Liu, A. L. M. Reddy, L. Ci, R. Vajtai, Q. Zhang, B. Wei, and P. M. Ajayan, Nat. Nanotechnol., 6 (2011) 496.

11. M. F. El-Kady, V. Strong, S. Dubin, and R. B. Kaner, Science, 335 (2012) 1326.

12. D. Zhan, Z. Ni, W. Chen, L. Sun, Z. Luo, Z. Lai, L. Lai, T. Yu, A. T. S. Wee, and Z. Shen, Carbon, 49 (2011) 1362.

13. M. A. Pimenta, G. Dresselhaus, M. S Dresselhaus, L. G. A. Cancado, A. Jorioa, and R. Saito, Phys. Chem. Chem. Phys., 9 (2007) 1276.

14. A. Watanabe and J. Cai, Proc. SPIE, 10520 (2018) 105200P.

15. C. Li, D. Li, J. Yang, X. Zeng, and W. Yuan, $J$. Nanomat., 2011 (2011) Article ID 319624, 1.

16. L. M. Malard, M.A. Pimenta, G. Dresselhaus, and M.S. Dresselhaus, Phys. Rep., 473 (2009) 51.

17. D. Graf, F. Molitor, K. Ensslin, C. Stampfer, A. Jungen, C. Hierold, and L. Wirtz, Nano Lett., 7 (2007) 238. 